\title{
Antipsychotics switching strategies in real life. A longitudinal study in clinical practice
}

\author{
Chris de Smidt* \\ Judith Haffmans ${ }^{*, * *}$ \\ Erik Hoencamp ${ }^{\star, \star *}$ \\ *Parnassia Bavo Groep, The Hague, \\ The Netherlands \\ ** Institute of Psychology, Leiden University, \\ Leiden, The Netherlands \\ THE NETHERLANDS
}

\begin{abstract}
Background and Objectives: Switching antipsychotics (APs) in the treatment of mental ilnesses such as schizophrenia is common practice for clinicians as well as a transitional moment associated with the possibility of adverse events and recurrence of psychoses. As in recent years, AP switching strategies have received more attention, a number of authors have recommended transitions with overlapping drug dosage regimens in time (such as tapering, cross-tapering, plateau switching) over abrupt switches. However, there is a paucity of data documenting how clinicians are switching APs in real life. Moreover, the question if recently recommended switching strategies are converted into everyday practice is still very much unanswered. The present investigation aims to study if indeed there is a preference for tapered approaches over abrupt switching.

Methods: In a retrospective longitudinal descriptive study, electronic prescription data from a large clinical psychiatric setting in the Netherlands were collected for the year 2007. Timelines of medication regimens were constructed for individual patients, enabling to identify transitions between APs. As patients could have been subjected to multiple switches in a given time period, transitions were expressed as episodes. Abrupt switches were defined as switches having no overlap in time.

Results: From a total of 5322 AP prescriptions involving 1465 patients, a total of 180 episodes (associated with 158 patients) were identified where APs were switched. Of these 180 episodes, $110(61 \%)$ involved abrupt transitions. 70 episodes (39\%) had overlap in time with an average taper length of 16.1 (SEM 1.6) days.

Conclusions: In the majority of cases in the studied clinical setting, APs are switched abruptly.
\end{abstract}

Received: 28 October 2010

Revised: 30 May 2011

Accepted: 31 May 2011 


\section{Introduction}

Switching antipsychotics is common practice during the treatment of psychosis as patient response and side effects remain not fully predictable. Although the concept of antipsychotic switching is common in clinical practice the CATIE study does not give much support for its efficacy ${ }^{1}$, the switching method itself might affect the succesful transition because of the possibility of adverse events and recurrence of psychoses. When confronted with a switch to a different AP, the prescribing doctor will have to address two questions: which AP will be next; and how will the switch be performed in the specific case? Abrupt switches, where the first AP is discontinued before the new AP is administered without an overlap in time, are simple, avoid the risk for confusion and decrease the risk of drug-drug interactions. Furthermore, they help to eliminate adverse effects of the first agent more rapidly ${ }^{2,3}$.

However, too rapid changes may be associated with the appearance of undesired physiological or psychiatric effects, decreasing the likelihood of a successful switch.

In order to improve successful switching, a variety of switching strategies have been proposed where the 2 agents had overlapping dosage regimens in time: tapering, cross-tapering, plateau-cross-tapering ${ }^{2-6}$, hybrid switches $^{5}$, plateau cross taper switches ${ }^{4,5}$, ascending / descending plateau switches ${ }^{2,6}$.

Data on the clinical value of the various switching approaches are sparse and conflicting. The metanalysis of Remington et al..$^{7}$ has been criticised as the four studies involved ${ }^{8-12}$ were industry-sponsored, favoring any switch outcomes $^{2}$. Recently, Pae et al. ${ }^{13}$ have documented support for the gradual switch to aripiprazole and other authors favour gradual switching approaches as well ${ }^{2-4,14}$.
Definite evidence of the superiority of general or AP-specific methods is not yet available ${ }^{15}$. However, an increasing number of authors now proposes gradual, overlapping switches opposed to abrupt changes ${ }^{4,5,16}$.

The choice for a switching approach is often based upon theoretical considerations rather than clinical evidence. APs with high cholinergic affinities as olanzapine and clozapine are preferably tapered to avoid cholinergic rebound ${ }^{14,15,17}$, and abrupt switching from high $\mathrm{H} 1$ affinity APs to low $\mathrm{H} 1$ affinity APs will result in rebound insomnia ${ }^{14}$. Switches from high affinity D2 APs (haloperidol, risperidone, ziprasidone) to low affinity D2 antagonists as quetiapine or clozapine are associated with the risk of dyskinesia or supersensitivity psychosis ${ }^{14}$, and tapered approaches have been recommended in order to avoid low D2 occupan$\operatorname{cies}^{14,17}$. On the other hand, the long halflife of AP depots provide a natural taper while the new drug is being introduced ${ }^{15}$.

In clinical practice, other types of considerations might predispose a health care provider to opt for a specific switch approach: the fear for possible medication errors might increase abrupt switching, availability of family surroundings, age, clinical setting or previous experience with switching approaches ${ }^{17}$.

In contrast to the increasing amount of articles containing proposals of switching approaches, surprisingly little is known about the reality of AP switching in clinical practice. As one of the few documents available, a retrospective study of 60 patients examined AP switching to amisulpride: $89 \%$ of these were switched to this agent without tapering ${ }^{17}$.

The present study aims to investigate real life AP switching approaches for all APs involved during a full year of AP pharmacotherapy in our hospital in the Netherlands. 
From a total of 5322 prescriptions involving a total of 1465 patients, a switching database was constructed enabling us to further analyse switching patterns between the various AP classes. As the study was longitudinal, timelines for all AP prescriptions could be constructed for every patiënt subjected to a AP switch. For all AP switching episodes, the switching type (abrupt versus overlapping strategies) was determined.

\section{Methods}

In a retrospective descriptive study, the prescription data of all patients receiving APs during the year 2007 in our hospital were analyzed. This hospital is a large psychiatric setting in The Hague, the Netherlands. All medication prescriptions are electronic, and provide detailed information on prescribed drug, formulation used, dosage, prescription time, prescribing doctors, departments amongst many other variables. All patients are included regardless of their pathological background, allowing to analyze if switching patterns vary between the various divisions. The set of electronic prescriptions for a given patiënt provides definitive and precise instructions for switching drugs and no further oral instructions are necessary per se.

The AP prescriptions were transferred to and analyzed in MS Access (version 2003). Those patients with presciptions for multiple APs during 2007 were identified, and medication timelines were constructed for individual patients (example shown in figure 1), enabling to map AP therapy during the year. As a patient could be subjected to multiple AP switches during the analyzed time period, switches and AP combinations were expressed as episodes.

Within MS Access, a separate table comprising all switching and combination episodes was constructed with details on the 1st and 2nd AP, divisions, duration of overlap, switch type, prescribing M.D. amongst other variables. An abrupt switch was defined as a transition of AP 1 to AP 2, having a maximum of 1 day overlap or a drug free period of maximally 3 days. The criterium of a maximum of 1 day overlap was chosen as doctors would occasionally make the electronic prescription a day in advance. In

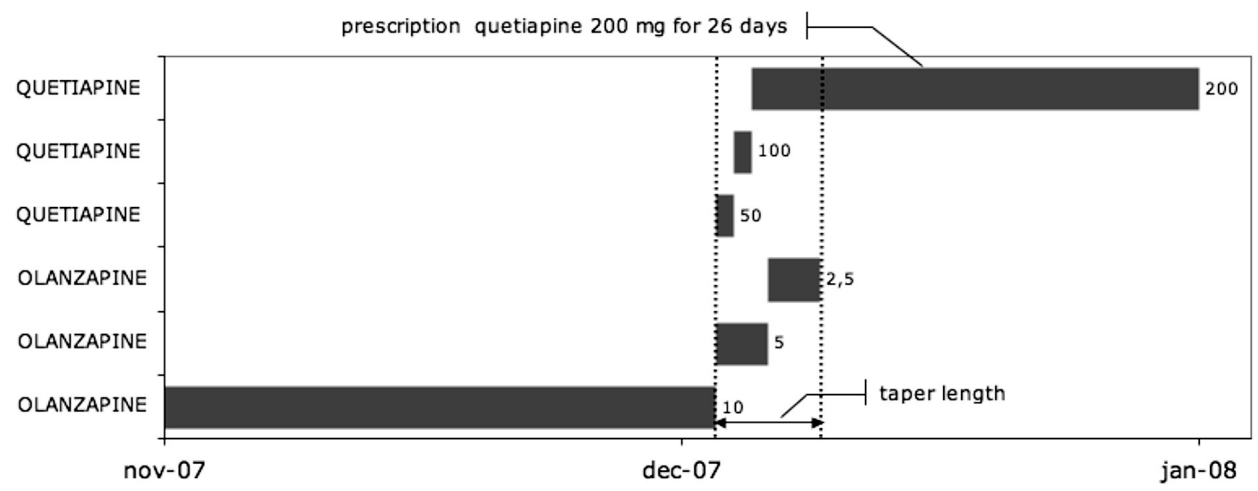

Figure 1. AP switching in clinical practice. In this example, a patient was switched from olanzapine to quetiapine by simultaneous dose decrease of olanzapine and dose increase of quetiapine (crosstapering). Total overlap in time of both agents (taper length) in this example was 7 days. 
almost all cases however, abrupt switches ocurred the following day. A 3-day cutoff was chosen as in two cases, a weekend had caused a delay where the psychiatrist, after being notified that the first AP had expired, had written the prescription the next monday. Abrupt switches could always be identified. On the other hand, there were some instances where cross-tapering could not be distinguished from other types of overlapping switches. For this reason, all overlapping switching approaches were pooled as "Tapered". With these definitions, possible dose decreases prior to the abrupt switch have no effects on the classification as the criterium was solely overlap in time.

Tapered switches were therefore defined as a transition between AP agents 1 and 2 having overlap in time of more than 1 day, in combination with a concurrent dose reduction of agent 1 , dose escalation of agent 2 , or both.

Combination therapies were defined as AP 1 and AP 2 having an overlap in time, but without concurrent dose reduction of agent 1 or dose buildup of agent 2 .

Switching patterns were, as a rule, apparent as the dose changes were documented. In specific cases, there was additional information in the electronic prescriptions detailing dose buildup or dose reduction schedules. Whenever the switch was not completely apparent (in 7 cases) the prescribing doctor was consulted in order to clarify the nature of the switch.

Relationships between switching methods, involved APs, prescribing M.D.s and other parameters: all queries were performed in MS Access utilizing standard procedures.

The total volume of prescriptions were calculated on a per prescription basis with the correct DDD (Defined Daily Dosis) value assigned to each drug preparation.

\section{Definitions:}

FGA: First Generation Antipsychotics ("classical antipsychotics", "typical antipsychotics") in this study were: benperidol, bromperidol, chlorpromazine, chlorprotixene, fluphenazine, flupentixol, fluspirilene, haloperidol, levomepromazine, penfluridol, perphenazine, periciazine, pimozide, pipamperone, thioridazine, tiapride, zuclopentixol.

$S G A$ : Second Generation Antipsychotics ("atypical antipsychotics") in this study were: aripiprazole, clozapine, quetiapine, olanzapine, risperidone, sertindole, sulpiride.

Taper length is defined as the total duration of overlap in time during a switch of APs in days (figure 1).

DDD: Defined Daily Dosis. The DDD is the assumed average maintenance dose per day for a drug used for its main indication in adults. Using DDD values enables comparison of different medicinal products. DDD values were calculated on a per prescription basis by dividing the total amount of mg prescribed per day by the DDD value of the specific preparation as provided by the WHO.

\section{Results}

During the studied period, a total of 5322 electronic prescriptions of APs were made in our hospital, associated with a total of 1465 patients that were treated with these agents. Prescription length could vary between 1 day and many months as residence time were highly variable and a subset of patients were permanently resident. The two most prescribed APs were clozapine and olanzapine. Table 1 represents, in descending order, the 15 most frequently prescribed APs, accounting for $97.6 \%$ of the prescribed AP volume. The majority $(62.5 \%)$ of pre- 
Table 1

Most frequently prescribed APs. For the year 2007, the total volume of prescriptions were calculated on a per prescription basis with the correct DDD value assigned to each drug preparation.

The indicated agents account for $97.6 \%$ of the prescribed AP volume

\begin{tabular}{lc} 
AP & DDDs (\%) \\
\hline Clozapine & 25,7 \\
Olanzapine & 20,1 \\
Zuclopenthixole (depot) & 9,9 \\
Quetiapine & 7,0 \\
Risperidone & 5,4 \\
Flupenthixole (depot) & 5,4 \\
Pipamperone & 4,6 \\
Flupenthixole & 4,0 \\
Haloperidol & 3,3 \\
Risperidone (depot) & 2,8 \\
Fluphenazine (depot) & 2,5 \\
Zuclopenthixole & 2,3 \\
Haloperidol (depot) & 2,1 \\
Aripiprazole & 1,4 \\
Penfluridol & 1,1 \\
\hline
\end{tabular}

scribed APs are SGA as compared to $37.5 \%$ FGAs. Of the FGAs, 55\% are depot formulations whereas much smaller proportions of the SGA $(4.5 \%)$ is prescribed as a depot (Risperdal Consta®).

The majority of the 1465 patients receiving APs were prescribed a single AP during the studied period. A total of 158 patients $(10.8 \%)$ was subjected to an AP switch in the year 2007. As patients sometimes had multiple switches or AP combinations, it was more useful to express and quantify switching as episodes.

The total number of switch episodes in this study added up to 180 , associated with 158 patients. The central results of the current study are shown in figure 2 and indicate that overall, the majority of AP switches $(61 \%)$ is abrupt, representing sudden transi- tions without time overlap. The total number of tapered episodes is 70, which corresponds to $39 \%$ of the total. As mentioned before, the various subtypes within the taper group could not always be identified. However, a first estimation indicated that about $50 \%$ of the tapered episodes are cross-tapered (simultaneous dose reduction of the first agent and dose buildup of the second agent, data not shown).

Figure 2 indicates that when comparing the AP classes, oral FGAs are subjected to abrupt switches more frequently than oral SGAs (69 vs 59\%). All 5 abrupt switches of FGA depots were to other FGA depots.

Of the 70 tapered episodes, the average length in time during which the transition took place (taper length) was 16.1 days (SEM 1.6), ranging from 2 to 58 (st. dev. 13.3) days. 

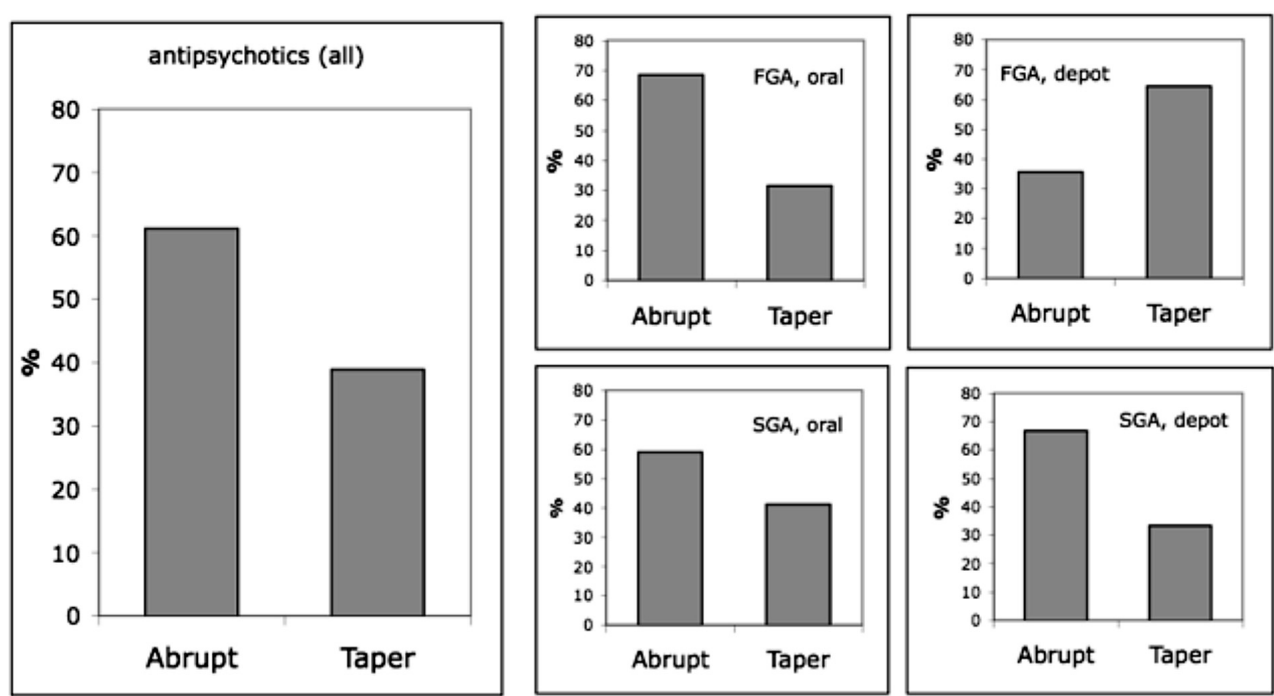

Figure 2. Switch type and first* AP. Associations of abrupt vs tapered switches in with the previously prescribed AP expressed as the $\%$ of total switching episodes. All antipsychotics: $\mathrm{n}=180$; oral FGA: $\mathrm{n}=73$; depot FGA: $\mathrm{n}=14$; oral SGA: 90; depot SGA: $\mathrm{n}=3$ ).

The FGA depots had an average taper length of a month, whereas the other agents were tapered approximately in a 2-week time span (table 2). Oral FGAs appeared to have been switched slightly more rapidly $(11.8 \pm 2.0$ days) than oral SGAs (15.3 \pm 2.1 days $)$.

When relating the switching frequency (irrespective of the method) to the relative volumes of the different AP classes, it could be estimated if FGA's were switched more frequently than SGAs (table 3). Whereas the total number of switches are roughly equal between FGAs and SGAs (87 vs 93), their prescribed volumes are not (37.5 vs $62.5 \%$ ). Table 3 indicates that when switching frequencies are corrected for their respective volumes, it appears that especially oral FGAs are switched more frequently than oral SGAs.

Table 2

Taper length per AP class

\begin{tabular}{lccc} 
First AP & Av. taper length $(\mathrm{d})$ & SEM & Number of episodes \\
\hline FGA oral & 11.8 & 2.0 & 23 \\
FGA depot & 30.6 & 4.8 & 9 \\
FGA all & 17.1 & 2.5 & 37 \\
SGA oral & 15.3 & 2.1 & 1 \\
SGA depot & n.a. & n.a. & 38 \\
SGA all & 15.2 & 2.1 & 70 \\
FGA \& SGA & 16.1 & 1.6 & 37 \\
\hline
\end{tabular}


Table 3

Total switches per AP class corrected for their prescribed volume

\begin{tabular}{lcccc} 
First agent & $\begin{array}{c}\text { Switch } \\
\text { Volume DDDs (\%) }\end{array}$ & Total switches (n) & Total switches (\%) & $\begin{array}{c}\text { Total switches } \\
\text { corrected (\%) }\end{array}$ \\
\hline FGA oral & 20.6 & 73 & 40.6 & 50.8 \\
FGA depot & 16.8 & 14 & 7.8 & 12.1 \\
FGA total & 37.5 & 87 & 48.3 & 62.9 \\
SGA Oral & 59.7 & 90 & 50 & 21.6 \\
SGA depot & 2.8 & 3 & 1.7 & 15.5 \\
SGA total & 62.5 & 93 & 51.7 & 37.1 \\
\hline All & 100 & 180 & 100 & 100 \\
\hline
\end{tabular}

\section{Discussion}

In spite of the fact that most recent publications and reviews ${ }^{2-4,14,16,18}$ recommend overlapping approaches when switching APs, this study shows that the majority $(61 \%)$ of switches are abrupt. When reviewing the literature on real life switching approaches, surprisingly few studies are available that document how prescribing M.D.s are switching APs in clinical practice. In a retrospective study of 60 patients switched to amisulpride, $89 \%$ of patients were switched abruptly ${ }^{17}$. For antidepressants, more data are available on switching patterns in clinical practice ${ }^{19}$, but to our knowledge, this is the first study of its kind that comprises the whole spectrum of prescribed APs in a real life situation.

The focus of this study was primarily on AP switching methods used in our hospital. We have classified the switches as Abrupt or Overlapping approaches ("Tapered"). The principal criterium was overlapping time in the presciption of the APs involved; abrupt switches had essentially no time overlap. Tapered approaches were characterized by simultaneous prescription in time with ei- ther dose reduction of the first agent, dose buildup of the second agent, or both (crosstapering). As there were some instances where cross-tapering could not be distinguished from other types of overlapping switches, all overlapping switching approaches were pooled as "Tapered". A limitation of this study is that among the abrupt switches, there may be also some episodes where the first agent was reduced in dose before changing to the next AP. This type of switching is associated with subtherapeutic AP levels and seems not to be common practice among MDs. Indeed, a survey in our database indicates that $<8 \%$ of abrupt switches is done with dose reduction prior to the switch (data not shown).

Since a number of years, all drug prescriptions made in our hospital are electronic and enable us to gather a complete and detailed overview of pharmacotherapy. We have constructed a database of 5322 prescriptions for APs in the year 2007, associated with a total of 1465 patients. Due to internal policies reducing AP polypharmacy, the majority of these patients were prescribed a single AP during this year, and a total of 270 (18.4\%) patients that had different APs in the year 2007 could be identified. 
In order to establish if the APs were associated with a switch or a combination, individual time-drug profiles (timelines) were constructed. As patients could be subjected to subjected to multiple AP switches during the analyzed time period, switches and AP combinations were expressed as episodes. By separating the episodes with AP combinations, a total number of patients with AP switches could be determined at $158(10.8 \%)$. A separate database was constructed with all episodes, detailing episode type (Abrupt, Tapered, Combination) as well as taper length and prescribing MD. True combinations of APs are currently subjected to a separate investigation (manuscript in preparation).

The central outcome of the present study is the large proportion of the abrupt switches in real life AP pharmacotherapy. Of the overlapping ("tapered") switches, the average taperlength was roughly two weeks and did not show major fluctuations between the various AP classes (table 2). One clear exception were FGA depots, having a taper length of a month.

Figure 2 shows the relationships between switchtypes and the AP that was discontinued (first AP) and indicates that there are no apparent associations with AP class. FGAs $(63.2 \%)$ and SGAs $(59.1 \%)$ all have abrupt switching frequencies around the average value of $61 \%$. One clear exception is that all FGA depot - FGA depot switches were abrupt (5 out of 5 episodes).

Most literature on AP switching focus on proposals ${ }^{3-5,16,20}$, study overall effectiveness of AP switching ${ }^{21}$ or study long term or short term effects and safety of switching methods $^{9,13}$. Whereas long term effects of switching methods appear to be small ${ }^{13}$, on the short term the switching method can possibly precipitate side effects or induce psy- chosis $^{3,4}$, affecting patient compliance and indirectly compromising long term success of AP therapy. As no final evidence is yet available for superiority of a single switching method, switching APs still remains an art rather than science. However, there appears to be a growing support for tapered switches in general ${ }^{4,5,16}$. For some specific agents as aripiprazol the switching method may be more critical than other agents, as is the early consensus in our own hospital.

Future directions on the analyses of switching strategies may involve underlying mechanisms such as the educational background of the MD's, their age, or switching strategies among their direct colleagues. It can be hypothesized that for some drugs, the personal influence of the MD has less impact on the switch than for antipsychotics that seem "easier to handle". Understanding the important underlying mechanisms for switching patterns would allow "diagnostics" of which variables to work on when optimizing switching strategies. Furthermore, the taper length is a variable that could be explored more profoundly. In this study, a taper length of roughly 2 weeks for oral APs is reported but the amount of data on taper length which is available in the literature is very scarce.

The intention of the current study was not to assess possible superiorities of switching strategies, but to investigate how the prescribing MD's are responding to the growing recommendations of tapered switching of antipsychotics.

To our knowledge, the present study documents, for the first time, that more than half of AP switches for the overall class of APs are abrupt. It is concluded that real life AP switching methods are still far away from the proposed guidelines. 


\section{Acknowledgements}

\author{
Mrs Samrah Khan is thanked for techni- \\ cal assistance.
}

\section{References}

1. Rosenheck RA, Davis S, Covell N, Essock S, Swartz $\mathrm{M}$, Stroup S, et al. Does switching to a new antipsychotic improve outcomes? Data from the CATIE Trial. Schizophr Res 2009; 107(1): 22-29.

2. Correll CU. Real-life switching strategies with second-generation antipsychotics. J Clin Psychiatry 2006; 67(1): 160-161.

3. Weiden PJ, Miller AL, Lambert TJ, Buckley PF. The art and science of switching antipsychotic medications, part 2. J Clin Psychiatry 2007; 68(1): e02.

4. Buckley PF, Correll CU. Strategies for dosing and switching antipsychotics for optimal clinical management. J Clin Psychiatry 2008; 69 Suppl 1: 4-17.

5. Lambert TJ. Switching antipsychotic therapy: what to expect and clinical strategies for improving therapeutic outcomes. J Clin Psychiatry 2007; 68 Suppl 6: 10-13.

6. Correll CU. From receptor pharmacology to improved outcomes: individualising the selection, dosing, and switching of antipsychotics. Eur Psychiatry 2010; 25 Suppl 2: S12-21.

7. Remington G, Chue P, Stip E, Kopala L, Girard T, Christensen B. The crossover approach to switching antipsychotics: what is the evidence? Schizophr Res 2005; 76(2-3): 267-272.

8. Casey DE, Carson WH, Saha AR, Liebeskind A, Ali MW, Jody D, et al. Switching patients to aripiprazole from other antipsychotic agents: a multicenter randomized study. Psychopharmacology (Berl) 2003; 166(4): 391-399.

9. Kinon BJ, Basson BR, Gilmore JA, Malcolm S, Stauffer VL. Strategies for switching from conventional antipsychotic drugs or risperidone to olanzapine. J Clin Psychiatry 2000; 61(11): 833-840.

10. Lee CT, Conde BJ, Mazlan M, Visanuyothin T, Wang A, Wong MM, et al. Switching to olanzapine from previous antipsychotics: a regional collaborative multicenter trial assessing 2 switching techniques in Asia Pacific. J Clin Psychiatry 2002; 63(7): 569-576.

11. Weiden PJ, Aquila R, Dalheim L, Standard JM. Switching antipsychotic medications. J Clin Psychiatry 1997; 58 Suppl 10: 63-72.
12. Weiden PJ, Simpson GM, Potkin SG, O’Sullivan RL. Effectiveness of switching to ziprasidone for stable but symptomatic outpatients with schizophrenia. J Clin Psychiatry 2003 ; 64(5): 580-588.

13. Pae CU, Serretti A, Chiesa A, Mandelli L, Lee C, Lee $\mathrm{C}$, et al. Immediate versus gradual suspension of previous treatments during switch to aripiprazole: results of a randomized, open label study. Eur Neuropsychopharmacol 2009; 19(8): 562-570.

14. Buckley PF. Receptor-binding profiles of antipsychotics: clinical strategies when switching between agents. J Clin Psychiatry 2007; 68 Suppl 6: 5-9.

15. Ganguli R. Rationale and strategies for switching antipsychotics. Am J Health Syst Pharm 2002; 59(22 Suppl 8): S22-26.

16. Weiden PJ, Buckley PF. Reducing the burden of side effects during long-term antipsychotic therapy: the role of "switching" medications. J Clin Psychiatry 2007; 68 Suppl 6: 14-23.

17. Burns T, Chabannes JP, Demyttenaere K. Switching Antipsychotic Medications: General Recommendations and Switching to Amisulpride. Curr Med Res Opin 2002; 18(4): 201-208.

18. Weiden PJ, Preskorn SH, Fahnestock PA, Carpenter D, Ross R, Docherty JP, et al. Translating the psychopharmacology of antipsychotics to individualized treatment for severe mental illness: a Roadmap. J Clin Psychiatry 2007; 68 Suppl 7: 1-48.

19. van Geffen EC, Hugtenburg JG, Heerdink ER, van Hulten RP, Egberts AC. Discontinuation symptoms in users of selective serotonin reuptake inhibitors in clinical practice: tapering versus abrupt discontinuation. Eur J Clin Pharmacol 2005; 61(4): 303-307.

20. Peuskens J. Switching approach in the management of schizophrenia patients. Int Clin Psychopharmacol 2000; 15 Suppl 4: S15-19.

21. Essock SM, Covell NH, Davis SM, Stroup TS, Rosenheck RA, Lieberman JA. Effectiveness of switching antipsychotic medications. Am J Psychiatry 2006; 163(12): 2090-2095.

Author for correspondence:

C. de Smidt PhD.

Parnassia Bavo Groep, Monsterseweg 83

the Hague, 2553 RJ, The Netherlands

Tel +31 703916624

Fax + 31703916612

E-mail: c.desmidt@parnassiabavogroep.nl 